
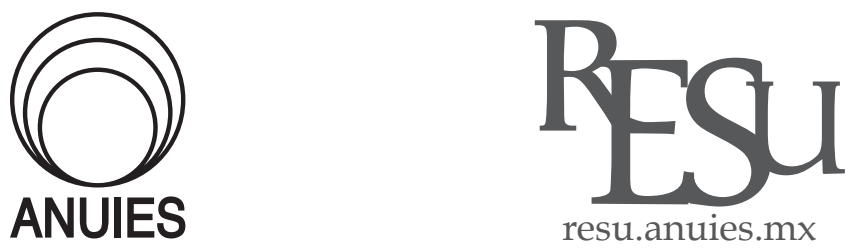

ARTíCULO

\title{
Factores que influyen en la preferencia por el tipo de estructura de liderazgo del máximo cuerpo colegiado de universidades iberoamericanas*
}

Factors influencing the preference for the type of leadership structure of the Supreme Collegiate Body of Ibero American universities Juan Abello Romero**, Claudio Mancilla-Mancilla y Francisco Ganga-Contreras

* Agradecimientos: Este trabajo está enmarcado dentro del proyecto de investigación "Formas de gobierno y modelos de valoración del desempeño universitario en Iberoamérica: Un estudio bajo un enfoque organizacional multiteórico", financiado por Conicyt (Proyecto Fondecyt Número 1161353).

**Universidad de los Lagos, Chile. Correo electrónico: jabello@ulagos.cl

Recibido el 2 de mayo del 2018; aceptado el 1 de marzo del 2019.

\section{Resumen}

Este trabajo muestra cómo influyen las características personales y el contexto organizacional en individuos que son parte de la comunidad académica de universidades iberoamericanas para preferir un tipo de estructura de liderazgo de los gobiernos universitarios, el basado en la teoría de agencia o en la teoría de servidores. El estudio se realiza en universidades donde actualmente el rector preside el Máximo Cuerpo Colegiado (MCC), es decir, el rector tiene un doble rol. Para alcanzar el objetivo del trabajo se realizan análisis no paramétricos y dos procedimientos de emparejamiento (matching) con aplicación de remuestreo (bootstraping) para tener resultados más precisos.

Palabras clave: Gobiernos corporativos universitarios; Máximos cuerpos colegiados; Estructura de liderazgo;

Universidades; Educación superior. 


\begin{abstract}
This paper shows how personal characteristics and organizational context influence individuals who are part of the academic community of Ibero American universities to choose a type of leadership structure of governments college students, based on agency theory or server theory. The study was made in universities where the currently rector also presides the Supreme Collegiate Body, that is, the rector has a double role. To achieve the work objective, nonparametric analyses and two matching procedures are performed with bootstraping to obtain more precise results.
\end{abstract}

Keywords: University corporative governments; Board of Directors; Leadership structure; Universities; Higher education.

\title{
Introducción
}

$\mathrm{E}_{\mathrm{r}}$ xiste una discusión en la literatura acerca de si en los gobiernos corporativos (GC) la existencia de dualidad (o el doble rol), que corresponde al hecho de que una misma persona presida el GC y ejecute las principales decisiones y estrategias dentro de la organización, es un factor que ayuda o no a un mejor desempeño de las organizaciones. Esta discusión es actualmente válida para instituciones como las universidades, donde es frecuente encontrar que los Máximos Cuerpos Colegiados (MCC), que son el gobierno corporativo de estas organizaciones (Ganga y Burotto, 2012), están presididos por el rector de dichas instituciones.

Por un lado, la teoría de agencia sugiere que es necesaria la separación de los roles, es decir que no exista dualidad, ya que se requiere ejercer un control para proteger a todas las partes interesadas de la organización. Por otra parte, la teoría de servidores no ve un conflicto en estos roles y percibe que los gerentes (el rector de una universidad en este trabajo) son suficientemente capaces, y que la combinación de los dos papeles fortalece la dirección y da poder al líder para tomar decisiones que demandan rapidez.

La mayoría de los expertos que pertenecen a universidades iberoamericanas ven el hecho de que quien preside el Mcc sea a su vez el rector podría interpretarse como problema (Ganga, 2016); desde lo anterior, es posible 
afirmar que prevalecería la teoría de agencia entre ellos. No obstante, hay diferencias relevantes de opiniones entre quienes actualmente tienen en sus universidades al rector presidiendo el MCC y entre quienes no tienen esa situación en sus instituciones. En estos últimos, son numericamente muy pocos quienes prefieren cambiar esa situación, es decir, cambiar de una estructura de liderazgo unitario a una dual; por otra parte, las opiniones son menos homogéneas entre quienes actualmente tienen al rector cumpliendo el doble rol, y en este sentido es posible identificar que existen grupos de individuos del cuerpo académico que sugieren mantener el liderazgo dual y otros que opinan debería ser diferente (cambiar a una estructura unitaria).

Entre otros elementos, las organizaciones están constituidas por personas que de acuerdo a sus proyecciones individuales, a su cercanía con el proceso de la toma de decisiones, características y actitudes, influyen y transforman a las instituciones, y a su vez estas últimas transforman a las personas. En este sentido, este trabajo postula que las preferencias de los expertos respecto de las teorías de agencia y servidores deben ser analizadas considerando las características tanto del cuerpo académico universitario como de las instituciones, porque algunas de ellas pueden influir (o no) sobre la elección del tipo de estructura del liderazgo del Mcc. En este sentido, percibir o no la dualidad como problema o fortaleza puede conducir a la transformación y la mejora en los niveles de eficiencia de las organizaciones.

Con base a los antecedentes anteriores, el objetivo de este trabajo es determinar qué factores condicionan la opinión de los expertos en la elección de la estructura de liderazgo de los gobiernos corporativos para las universidades. Para alcanzarlo se hace uso de técnicas de análisis no paramétricos, como prueba Kruskal-Wallis y análisis de emparejamiento de datos (matching). Los principales resultados mostraron que efectivamente existen factores que condicionan la preferencia por un tipo de estructura de liderazgo en los MCc de universidades iberoamericanas.

Un objetivo anhelado de larga data es elevar los niveles de desarrollo y bienestar de la sociedad, y que además es postulado desde diversas disciplinas y creencias. En este contexto, estudiar a las universidades y a sus gobiernos corporativos, siendo elementos que contribuyen a los propósitos anteriores, muestra ser relevante para plantearse el mejoramiento del desempeño en la formación y la generación de conocimiento de las universidades, como también hacer un uso más adecuado de los recursos destinados y utilizados en ellas. 
La siguiente sección proporciona un marco teórico sobre el que se basa el presente análisis. En secciones posteriores se proporcionan el material y el método, luego los resultados, para finalizar con las conclusiones.

\section{Marco Teórico}

La estructura del liderazgo del directorio (en nuestro caso los MCc universitarios) es un potente mecanismo de gobierno corporativo representado por las posiciones de su presidente o por el máximo ejecutivo, ya sea el gerente en una empresa o el Rector en una universidad. Si el presidente del consejo directivo y el rector son distintas personas, se habla de una estructura de liderazgo unitario; $y$, si el mismo individuo ocupa las dos posiciones se indica que es una estructura dual (Adrian, Wright y Kilgore, 2016; Briano-Turrent y Saavedra-García, 2015; Christensen, Kent, Routledge y Stewart, 2015).

La estructura del liderazgo de los directorios tiene especial importancia en aspectos como el desempeño de las organizaciones, la transparencia, la divulgación de información y la gestión de las firmas, entre otros aspectos, y es por ello que ha sido materia de estudio en los planos teórico y empírico (Acar y Ozkan, 2017; Alfraih, 2016; Arora y Sharma, 2016; Carmona, De-Fuentes y Ruiz, 2016; Duru, Raghavan y Zampelli, 2016; Lai y Tam, 2017; Krause, 2017; Nas y Kalaycioglu, 2016; Petrou y Procopiou, 2016; Qian, Wang, Geng, y Yu, 2017; Rahman y Hamdam, 2017; Rao y Tilt, 2016; Sheikh y Khan, 2016; Titova, 2016; Torchia y Calabro, 2016; Tang, 2017; Villanueva-Villar, Rivo-López, y Lago-Peñas, 2016; Withers y Fitza, 2017). En el plano teórico, dos teorías se disputan la explicación del tipo de estructura del liderazgo; las diferencias argumentales entre ambos enfoques radican en el rol prioritario que juegan los directorios en una organización. Por un lado, la teoría de agencia postula la separación de la función del máximo ejecutivo con la del presidente del directorio; esto está sustentado en los roles que le da esta teoría a los directorios, que son organismos que deben ejercer el monitoreo del desempeño del máximo ejecutivo y su equipo con el propósito de proteger los derechos de las partes interesadas ante la probable existencia del problema de agencia (Appuhami y Bhuyan, 2015; Amran, Periasamy, y Zulkafli, 2014; Ashwin, Krishnan y Rejie, 2015; Broye, Francois, y Moulin, 2017; Chancharat, Krishnamurti y Tian, 2012; Dalton y Aguinis, 2013; Das y Dey, 2016; Elsayed, 2010; Fama, 1980; Ganga y Maluk, 2016; Gao y Jain, 2012; Gaur, Bathula y Singh, 2015; Perez-Calero, Guerrero-Villegas y Hurtado, 2017; Rodrigues, 2017; 
Schepker y Oh, 2013; Taniman y Shannassy, 2015; Tien, Chen, y Chuang, 2013; Veprauskaite y Adams, 2013).

Es decir, en el caso del gobierno corporativo universitario, esta teoría postula que el rector no debería presidir el MCC, y este último debería velar porque todos los intereses estén resguardados. Por lo tanto, se asume que aquellos expertos que prefieren una estructura de liderazgo unitario privilegian la teoría de agencia.

Por otra parte, la teoría de servidores tiene una posición relajada en cuanto a la dualidad, ya que considera al administrador como una persona que se ordena bajo una idea colectivista y pro-organizativa (Eddleston, Kellermanns, y Zellweger, 2012), y por lo tanto asume que los gerentes son intrínsecamente dignos de confianza y son buenos administradores; ellos están conscientes, según la teoría, que es su deber salvaguardar los recursos de la empresa y lograr el mejor desempeño (Davis, Schoorman y Donaldson, 1997; Gaur et al., 2015). La teoría de servidores sostiene que el directorio debe tener un rol de apoyo o asesoramiento con el poder ejecutivo de las firmas, y esto se materializaría correctamente si la estructura del liderazgo fuese dual; además permite afrontar con mayor rapidez los problemas, y asimismo elimina la posibilidad de rivalidad entre el presidente y el máximo directivo y con ello agiliza la toma de decisiones y disminuye los costos de transferencia de información (Guillet, Seo, Kucukusta, y Lee, 2013; Pham, Oh y Pech, 2015; Wang, Sun, Yu, y Zhang, 2014). Bajo esta teoría, un CEO-Presidente siente satisfacción profesional, personal y social por asumir una doble función (Taniman y Shannassy, 2015), además esto puede ser propio de máximos ejecutivos fundadores (Gao y Jain, 2012). De esta manera, en un gobierno corporativo universitario, esta teoría no vería un conflicto en que el rector sea la persona que presida el MCC, y este último debería asesorar y apoyar la gestión del rector de turno.

En todo caso, a la luz de los antecedentes teóricos, parece razonable considerar a las teorías de agencia y servidores como complementarias, ya que cada una colabora en la construcción completa de la imagen de la estructura de liderazgo y el rol de los gobiernos corporativos (Elsayed, 2010). En otras palabras, cada estilo de estructura de liderazgo del MCC basado en estos enfoques representaría un trade-off, una suerte de compensación, entre sus distintas fortalezas y debilidades. En el siguiente esquema (figura 1) se reflejan las diferencias entre ambas teorías y aspectos hasta aquí tratados. 
Figura 1

Teorías que sostienen las estructura de liderazgo y el rol de los gobiernos corporativos

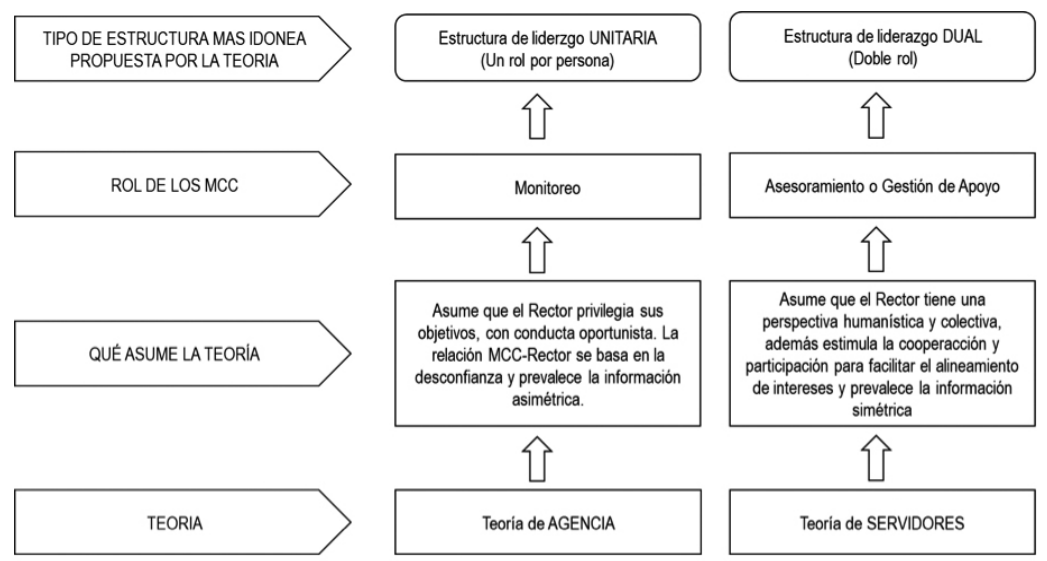

Fuente: elaboración propia.

De acuerdo a (Ganga, 2016), en relación a los gobiernos corporativos universitarios, mayoritariamente profesores o académicos que trabajan en universidades latinamericanas opinan que quien debería presidir el Mcc debe ser alguien diferente al rector, lo que nos indica que es la teoría de agencia, la cual busca resguardar a todas las partes interesadas y ve un conflicto importante en la existencia del doble rol, la prevaleciente entre los expertos. Sin embargo, el análisis comienza a mostrar importantes diferencias cuando se separa a quienes tienen actualmente en sus instituciones al rector presidiendo el Mcc de quienes no. En este último grupo, son proporcionamente muy pocos quienes desearían cambiar a una estructura de liderazgo dual, mientras que entre quienes actualmente tienen una estructura dual en sus MCc, un importante grupo prefiere cambiar esa situación, y como es de suponer, hay otro que prefiere mantenerla. Al analizar las características de los individuos, se observa que algunas de ellas podrían justificar la preferencia por un tipo de estructura de liderazgo, y diversos autores puede contribuir a ayudar a explicar la influencia de estas características.

Por ejemplo, Ruiz (1995) entrega la idea de que los directivos, quienes toman las decisiones (quienes ejercen el poder) podrían tender más a aumentar los controles y supervisión de las organizaciones que a responder efectivamente a las necesidades del entorno. Desde esta perspectiva, se podría indi- 
car que los directivos de las universidades podrían eventualmente estar más preocupados por la organización misma, que por responder tanto al entorno interno como externo. En el sentido anterior, la eficiencia de la institución se reduciría, lo que vendría a apoyar la teoría de agencia en el sentido de la necesidad de ejercer un control sobre los directivos.

Del otro lado, el proceso de toma de decisiones a veces se ve dificultado por la existencia de barreras para ejercer la autoridad, lo cual implica que no habría suficiente poder (para tomar decisiones); así, algunos buenos administradores ven en el poder una herramienta para hacer a las organizaciones más eficientes y productivas, y Pfeffer (1993) entiende la pugna gerencial (ejercicio o uso del poder) como una potencial fuerza. En esta investigación se considerará, en cambio, como la posibilidad de tomar decisiones en las universidades. No obstante, el mismo autor hace referencia a trabajos donde se muestra la ambigüedad de las personas frente al concepto "poder", en el sentido de que si bien se valora la posibilidad de ejercerlo y es entendido como un símbolo de éxito, también se reconoce que limita la eficiencia de las organizaciones (p. 13-14).

Contextualizado este trabajo, la anterior ambigüedad hace reflexionar acerca de las personas que son parte de las universidades, quienes pueden tener diferentes visiones respecto al ejercicio de la toma de decisiones (poder), de tal forma que mientras estén más cercanos a este proceso, debería existir una mayor preferencia por tener atribuciones para ejercerlo (es decir, una mayor preferencia por la teoría de servidores). Así, existirán otros individuos que tengan preferencias por la preponderancia de un mayor control sobre el proceso de la toma de decisiones en los gobiernos corporativos universitarios (es decir, mayor preferencia por la teoría de agencia).

Zarco, Rodriguez y Martón (2004) indican que tanto las organizaciones como los individuos se construyen y transforman mutuamente (p. 43), y si bien el comportamiento de las personas es condicionado por el contexto organizacional, también las características personales de los individuos y su comportamiento pueden transformar a las instituciones. Por otra parte, Kolb, Rubin y McIntyre (1980) proponen que los individuos, según sus aspiraciones y etapa de la carrera profesional, podrán tener distintas pretensiones para influir o no en las organizaciones. Esto conduce a reforzar la reflexión que ciertas circunstancias podrían influir sobre la percepción de la toma de decisión (poder). Así, por ejemplo, se podría pensar en que el cuerpo académico que esté pronto al retiro puede tender a estar más predispuesto a aceptar una estructura donde exista dualidad, tanto por su jerarquía y la cercanía en la toma de decisiones, o también puede no interesarle tal dualidad 
justamente porque en un periodo cercano dejará de tener la posibilidades de tener influencia al interior de la organización.

Si bien en el párrafo precedente se desliza como ejemplo la idea de que la edad puede ser un factor que influya sobre la percepción de la dualidad, se propone, tomando lo indicado por Zarco et al. (2004), que otras características personales y las condiciones organizacionales también pueden hacerlo sobre el doble rol del rector. Específicamente, se propone que la edad, el género, la formación de postgrado (características personales), la experiencia universitaria, el cargo jerárquico en ejercicio y el tipo de universidad (como el contexto organizacional que influye sobre los individuos), son elementos que pueden ayudar a entender cómo los individuos pueden tener una mayor o menor preferencia por estructuras de liderazgo de los MCC basado en las teorías de agencia o servidores. Así, nuestra hipótesis de trabajo es: las comunidades universitarias prefieren estructuras de liderazgo unitario o dual de los MCC (prevelencia de la teoría de Agencia o de Servidores) condicionados por las características personales de los individios y las del entorno organizacional.

A juicio de los investigadores, esto va adecuadamente alineado a lo que se esperaría de personas que trabajan en instituciones como son las universidades, por cuanto éstas son organizaciones que deberían ser por excelencia lugares de reflexión y crítica, y que además se exija transparencia en diversas dimensiones.

Tal como se indicará en la siguiente sección, la selección de los factores anteriormente individualizados responde tanto a la revisión hasta aquí hecha como a la disponibilidad de información para realizar el presente trabajo.

\section{Materiales y método}

Para abordar el objetivo del trabajo se hace uso de una base de datos generada por el Proyecto de Investigación denominado Sistemas de Gobierno de las universidades iberoamericanas: un análisis desde la teoría de agencia (Proyecto Fondecyt 1131134, Chile), que corresponde a un cuestionario denominado "Estudio sobre gobierno universitario" y fue aplicado durante los años 2014-2016 a 854 expertos de 19 países (18 países latinoamericanos más España) pertenecientes a más de 200 universidades. Se extrajeron preguntas que se agruparon en dimensiones relacionadas con las características personales, como las del entorno organizacional. Las preguntas que fueron utilizadas para el propósito de este trabajo son entregadas en el Anexo 1.

Del total de observaciones sólo se consideraron aquellos expertos en los que en su universidad actualmente quien preside el MCC es el rector, de tal 
forma que sólo se contemplaron 631 observaciones. No obstante, tal como se detallará más adelante, a medida que se van realizando las pruebas estadísticas, se eliminan observaciones que se consideraron perdidas por cuanto no cuentan con respuestas en todas las variables de interés. Así, las variables que se utilizaron en este trabajo son las que se detallan a continuación en la tabla 1:

Tabla 1

Variables utilizadas

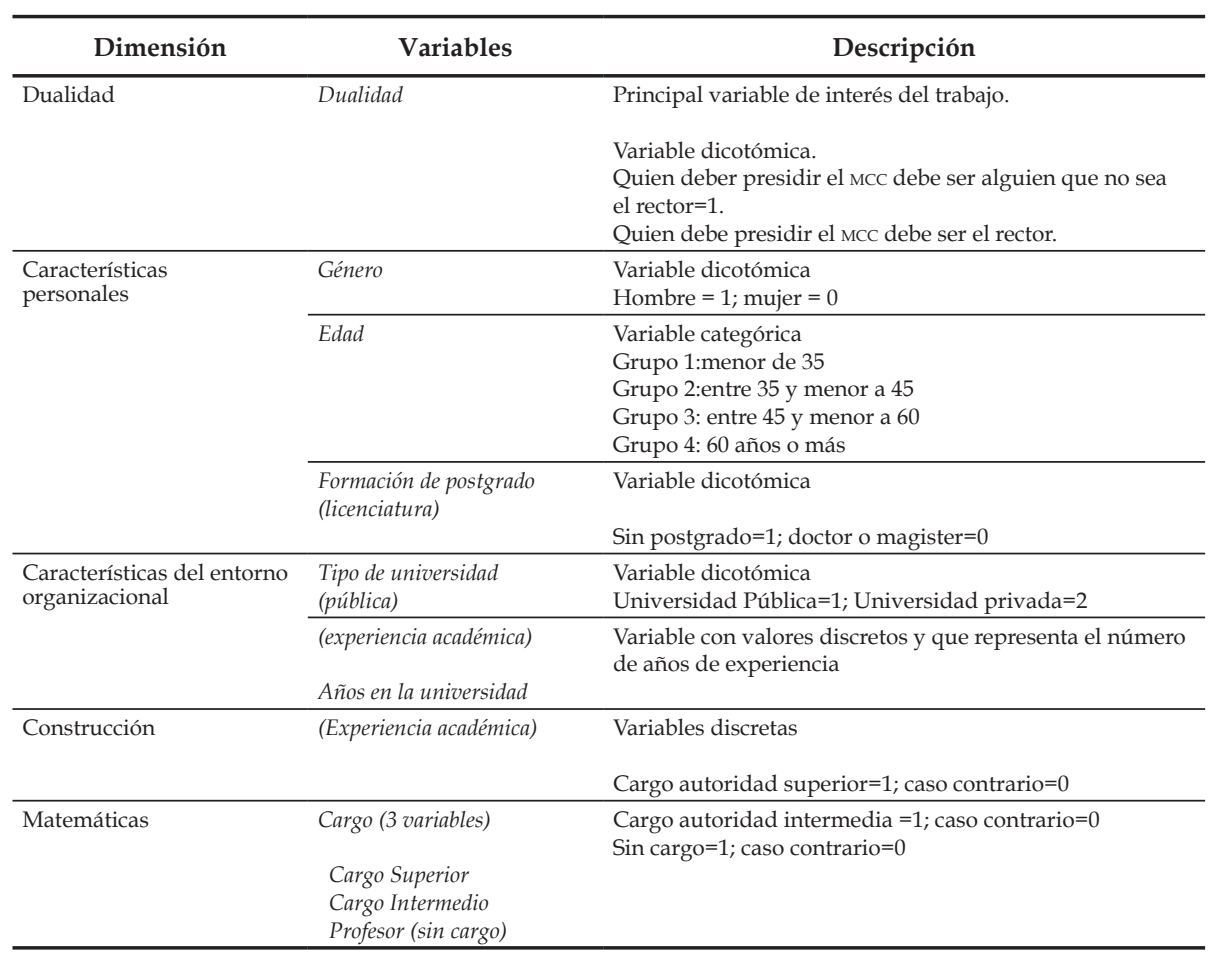

Fuente: Elaboración propia, basado en cuestionario Estudio sobre gobierno universitario (2014-2016).

Para trabajar los datos se utilizaron dos métodos. El primero de ellos consistió en analizar las diferencias de medias utilizando la prueba de U-Mann Whitney (análisis para dos grupos) y la generalización de éste por medio del test del Kruskal Wallis (para tres grupos o más). La naturaleza de los datos y las respuestas (contar con variables dicotómicas por ejemplo) hizo que haya sido necesario utilizar estas pruebas por cuanto no todas las variables se distribuyen estadísticamente como una normal (Levin y Rubin, 2010).

La interpretación del test U-Mann Whitney es bastante más simple e indica que si se rechaza la hipótesis estadística de igualdad de dos grupos, nos se- 
ñala que el resultado de la variable analizada entre (por ejemplo) expertos de universidades públicas y privadas es significativamente diferente. En el caso de que existan 3 o más grupos (aquí se aplica la prueba de Kruskal Wallis), el que se rechace la hipótesis estadística nula significa que al menos uno de los grupos es diferente al resto en relación al valor observado para una variable.

Sin embargo, para proveer un análisis estadísticamente más robusto se estudia la influencia de las dimensiones antes indicadas sobre la preferencia por el tipo de estructura de liderazgo, por medio de la aplicación de dos tipos de emparejamiento de datos (matching). Con esta técnica se compara a dos grupos de individuos que sean lo más similares en sus características (que serán las variables de control en el proceso de emparejamiento), pero cuya diferencia de percepción de que la dualidad es un problema esté explicada por otra variable, como lo será el hecho de que por ejemplo el experto tenga o no postgrado. Como el resultado es una variable dicotómica (la dualidad es un problema con valor 1 , o no es un problema con valor 0 ), al comparar el promedio de la variable de un grupo con el otro se puede interpretar también como una probabilidad. Adicionalmente, como se están comparando dos grupos gemelos, el foco del análisis es determinar si existen o no diferencias significativas entre ellos.

Por ejemplo, dado un grupo de características, un experto que sea de una universidad pública, ¿tiene una mayor probabilidad de preferir una estructura de liderazgo unitaria (ausencia de dualidad)? En principio, no se podría responder directamente esta pregunta porque no hay capacidad de identificar el comportamiento de una persona que fue señalada como de una universidad pública, como si fuese un experto que pertenece a una universidad privada.

La manera de resolver la pregunta anterior, es construir un grupo de tratamiento (o grupo de interés) constituido por aquellas personas que son de universidades públicas, y otro de expertos que son de universidades privadas. Luego las observaciones de cada grupo se emparejan con otra(s) del otro grupo que sean lo más similares posibles (como si fuesen gemelos) en las demás variables de control, exceptuando (siguiendo con nuestro ejemplo) al hecho del tipo de universidad a la que pertenecen.

Por ejemplo, en una situación simplificada, si una observación es de un experto que pertenece a una universidad pública, que es hombre y además tiene postgrado, se busca por medio de la técnica a otro experto que también sea hombre y con postgrado, pero que pertenezca a una universidad privada. El proceso, que se repite para las demás observaciones, hace que se pierdan algunas de éstas por cuanto no todas tienen un "gemelo aproximado" o no 
son parte del soporte estadístico común. Una vez construidos estos grupos gemelos por medio del proceso de emparejamiento, se comparará la percepción del problema de dualidad de cada uno de ellos.

Existen diversos métodos de emparejamiento, por ejemplo están los basados en el propensity score, que consiste en calcular un puntaje para cada observación, y luego se empareja la observación del grupo de tratamiento con la del grupo de control con el puntaje más parecido (en este caso escogeremos el vecino más cercano); o el procedimiento de emparejamiento que consiste en calcular la distancia vectorial de Mahalanobis, que consiste en el cálculo de dicha distancia y se empareja una observación con la menor distancia vectorial del otro grupo. Independiente de los procedimientos, una de las ventajas más relevantes de usar métodos de matching, es que no se requiere asumir ningún tipo de función para estimar el impacto de alguna variable (Wagner, 2007). Este último investigador estudió cómo influyen variables personales sobre la decisión de crear una empresa en Alemania por medio de estos métodos.

Finalmente se optó en este trabajo, para cada uno de los procedimientos de emparejamiento, un procedimiento bootstraping (remuestreo), que permite determinar errores estándar e intervalos de confianza de forma más precisa (Efron y Tibshirani, 1986).

\section{Resultados y discusión}

Los resultados obtenidos una vez aplicadas las metodologías adoptadas se encuentran en las tablas 2 y 3 . Desde la tabla 2 (donde se analizan las diferencias de las medias por grupos por medio de las pruebas U Mann-Whitey y Kruskal-Wallis) se puede observar que el 55,31\% de los expertos es crítico con respecto a la estructura de liderazgo del tipo dual en las universidades. En este sentido se observa que la teoría de agencia es la que prevalecería dentro de los expertos. Las diferencias, según las características utilizadas en este trabajo, varían e influyen significativamente sobre la preferencia, actitud crítica o percepción del problema.

No obstante lo anterior, se debe recordar un hallazgo no tratado en este trabajo, y es que el hecho de que el cuerpo académico que actualmente tiene una estructura de liderazgo unitario (basado en la teoría de agencia) en su universidad, tan sólo el 2,91\% preferiría cambiar a una estructura de liderazgo dual, lo que contrasta fuertemente con el grupo estudiado en esta investigación. Lo anterior, conduce a plantear como desafíos futuros el definir 
cuál sería el tipo de estructura de liderazgo en los MCc más adecuado para las universidades.

Es así como en el caso del sexo se observa que los hombres tenderían (aunque con un nivel de significancia cercana al 10\%) a ser más críticos con la existencia del doble rol. No obstante, como se puede apreciar en la tabla 3, por medio de los procedimientos de emparejamiento (comparación de grupos gemelos) no existen tales diferencias y se desecharía que el sexo influya sobre la percepción.

En el caso de la edad, se pueden observar dos hechos. Por una parte, la preferencia por el tipo de estructura de liderazgo está influenciado según el grupo etario al que pertenezcan los expertos. El segundo aspecto que se puede observar (tabla 2), es que se puede inferir la existencia de una U invertida, ya que el grupo más joven (menores de 35 años), y el grupo de más edad (grupo de más de 65 años), son menos críticos con la presencia del doble rol en comparación al grupo de edad intermedia (entre 35 y 65 años). Dado esto, para el proceso de emparejamiento de datos, se aglomeraron los más jóvenes y los más adultos en un grupo, y los de mediana edad en otro; al respecto, se puede observar en la tabla 3 que la diferencia entre ambos es muy significativa, llegando a concluir que los expertos de mediana edad (los que en apariencia son personas más activas y productivas dentro de las organizaciones) son un $47,37 \%$ más críticos con la existencia de la dualidad que sus pares de menor y mayor edad.

Independiente del método adoptado, se observa claramente que expertos situados en escalas superiores de la organización de las universidades (es decir, más cercanas al rector, y que son directivos de las instituciones) tienden a preferir que siga siendo el rector quien presida el MCC, por sobre lo que opinan expertos que no ocupan posiciones jerárquicas superiores. Por medio del emparejamiento de datos (tabla 3), se determina que los expertos sin cargos son un $41,38 \%$ más críticos (con un nivel de significancia del 1\%) que sus pares con cargos intermedios y superiores en el sentido de preferir que la estructura del liderazgo del Mcc no sea dual.

En relación al tipo de universidad, se observa que aunque por medio del procedimiento de emparejamiento de propensity score, el contexto organizacional de las universidades públicas muestra tener importancia; su significancia es cercana al 10\% y en general los otros métodos desechan que esta variable sea relevante.

Desde el lado de la formación, se determina que expertos con grados académicos superiores (de maestría y doctorado), es decir, expertos situados en 
escalas superiores de la generación o transmisión del conocimiento, tienden a preferir un tipo de estructura de liderazgo unitario: dicho de otro modo, el cuerpo académico sin formación de postgrado es menos crítico con estructura dual de los MCC.

Finalmente, el tiempo de experiencia universitaria refleja, por medio de todos los métodos adoptados, que influye sobre la preferencia por el tipo de estructura de liderazgo. Es así como los individuos con más experiencia, organizacionalmente hablando, prefieren que el rector no presida el MCC.

Tabla 2

Descriptivo y resultado test U-Mann Whitney - Kruskal Wallis

\begin{tabular}{|c|c|c|c|c|c|}
\hline & Variable & Promedio & $\begin{array}{c}\text { Desviación } \\
\text { estándar }\end{array}$ & $\begin{array}{c}\text { Número de } \\
\text { observaciones }\end{array}$ & P valor \\
\hline $\begin{array}{l}\text { Problema } \\
\text { Dualidad }\end{array}$ & Dualidad & 0.5531 & 0.4976 & 631 & -- \\
\hline \multirow{2}{*}{ Género } & Hombre & 0.5792 & 0.4943 & 404 & \multirow{2}{*}{0.0912 * } \\
\hline & Mujer & 0.5090 & 0.5010 & 222 & \\
\hline \multirow{4}{*}{ Edad } & Menor de 35 & 0.5000 & 0.5058 & 44 & \multirow{4}{*}{$0.0153 * *$} \\
\hline & Entre 35 y menor a 45 & 0.6047 & 0.4904 & 172 & \\
\hline & Entre 45 y menor a 60 & 0.5888 & 0.4928 & 321 & \\
\hline & 60 años o más & 0.3780 & 0.4879 & 82 & \\
\hline \multirow{3}{*}{ Cargo } & Autoridad superior & 0.0938 & 0.2938 & 64 & \multirow{3}{*}{$0.0001 * * *$} \\
\hline & Autoridad intermedia & 0.3672 & 0.4834 & 177 & \\
\hline & Sin cargo & 0.7620 & 0.4264 & 353 & \\
\hline \multirow{2}{*}{$\begin{array}{l}\text { Tipo } \\
\text { Universidad }\end{array}$} & Universidad Pública & 0.5626 & 0.4966 & 503 & \multirow{2}{*}{0.3912} \\
\hline & Universidad Privada & 0.5200 & 0.5016 & 125 & \\
\hline \multirow{2}{*}{$\begin{array}{l}\text { Grado } \\
\text { académico }\end{array}$} & Solo licenciatura & 0.4896 & 0.5009 & 241 & \multirow{2}{*}{$0.0061 * * *$} \\
\hline & Magister o doctor & 0.6021 & 0.4901 & 377 & \\
\hline \multirow{2}{*}{$\begin{array}{l}\text { Años en la } \\
\text { universidad }\end{array}$} & $\begin{array}{l}\text { Bajo mediana años } \\
\text { universidad }\end{array}$ & 0.5038 & 0.5009 & 264 & \multirow{2}{*}{$0.0121 * *$} \\
\hline & $\begin{array}{l}\text { Sobre mediana años en } \\
\text { universidad }\end{array}$ & 0.6119 & 0.4882 & 268 & \\
\hline
\end{tabular}

Fuente: elaboración propia.

***, **, * indican nivel de significación del 1\%,5\% y $10 \%$ respectivamente. 
Tabla 3

Resultado emparejamiento de datos - Matching

\begin{tabular}{|c|c|c|c|c|c|}
\hline \multirow{2}{*}{ Variables } & \multirow{2}{*}{$\begin{array}{l}\text { Identificación grupo de } \\
\text { tratamiento y control }\end{array}$} & \multicolumn{2}{|c|}{$\begin{array}{c}\text { Propensity Score } \\
\text { (vecinos más cercanos) }\end{array}$} & \multicolumn{2}{|c|}{ Mahalanobis Distance } \\
\hline & & $\begin{array}{c}\text { Coeficiente } \\
\text { (probabilidad) }\end{array}$ & $\begin{array}{c}\text { Error } \\
\text { estándar }\end{array}$ & $\begin{array}{c}\text { Coeficiente } \\
\text { (probabilidad) }\end{array}$ & $\begin{array}{c}\text { Error } \\
\text { estándar }\end{array}$ \\
\hline \multirow[t]{2}{*}{ Género } & $\begin{array}{l}\text { Grupo de tratamiento: } \\
\text { Hombre }\end{array}$ & \multirow[t]{2}{*}{-0.0281} & \multirow[t]{2}{*}{0.0778} & \multirow[t]{2}{*}{0.0557} & \multirow[t]{2}{*}{0.0685} \\
\hline & Grupo Control: Mujer & & & & \\
\hline \multirow{2}{*}{$\begin{array}{l}\text { Edad (grupo } \\
\text { joven y } \\
\text { adulto, } \\
\text { versus grupo } \\
\text { intermedio) } \\
\text { Grupo } 1 \text { y } 4 \text {, } \\
\text { versus grupo } \\
2 \text { y } 3\end{array}$} & $\begin{array}{l}\text { Grupo de tratamiento: } \\
\text { Grupo etario Menor y } \\
\text { mayor edad }\end{array}$ & \multirow[b]{2}{*}{-0.4737} & \multirow[b]{2}{*}{$0.1871^{* *}$} & \multirow[b]{2}{*}{-0.2553} & \multirow[b]{2}{*}{$0.0681^{* * *}$} \\
\hline & Grupo Control: Mujer & & & & \\
\hline \multirow{6}{*}{ Cargo } & $\begin{array}{l}\text { Grupo de tratamiento: Cargo } \\
\text { superior }\end{array}$ & \multirow[t]{2}{*}{-0.3750} & \multirow[t]{2}{*}{$0.1126^{* * *}$} & \multirow[t]{2}{*}{-0.4821} & \multirow[t]{2}{*}{$0.1193^{* * *}$} \\
\hline & Grupo Control: Otros cargos & & & & \\
\hline & $\begin{array}{l}\text { Grupo de tratamiento: Cargo } \\
\text { intermedio }\end{array}$ & \multirow[t]{2}{*}{-0.2857} & \multirow[t]{2}{*}{$0.0913^{* * *}$} & \multirow[t]{2}{*}{-0.2208} & \multirow[t]{2}{*}{$0.0771^{* * *}$} \\
\hline & Grupo Control: Otros cargos & & & & \\
\hline & $\begin{array}{l}\text { Grupo de tratamiento: Sin } \\
\text { cargo }\end{array}$ & 0.4138 & $0.0633^{* * *}$ & 0.4178 & $0.0645^{\star * *}$ \\
\hline & Grupo Control: Otros Cargos & & & & \\
\hline \multirow{2}{*}{$\begin{array}{l}\text { Universidad } \\
\text { pública }\end{array}$} & $\begin{array}{l}\text { Grupo de tratamiento: } \\
\text { Universidad Pública }\end{array}$ & \multirow{2}{*}{-0.1157} & \multirow{2}{*}{$0.0626^{*}$} & \multirow{2}{*}{-0.0814} & \multirow{2}{*}{0.0562} \\
\hline & $\begin{array}{l}\text { Grupo Control: Universidad } \\
\text { Privada }\end{array}$ & & & & \\
\hline \multirow{2}{*}{$\begin{array}{l}\text { Grado } \\
\text { académico }\end{array}$} & $\begin{array}{l}\text { Grupo de tratamiento: Solo } \\
\text { tratamiento }\end{array}$ & \multirow{2}{*}{-0.1905} & \multirow{2}{*}{$0.0903^{* *}$} & \multirow{2}{*}{-0.1164} & \multirow{2}{*}{$0.0654^{*}$} \\
\hline & $\begin{array}{l}\text { Grupo Control: Magister y/o } \\
\text { doctor }\end{array}$ & & & & \\
\hline \multirow{2}{*}{$\begin{array}{l}\text { Años de } \\
\text { experiencia } \\
\text { en la } \\
\text { universidad } \\
\text { (Bajo } \\
\text { mediana } \\
=\text { Menor } \\
\text { experiencia) }\end{array}$} & $\begin{array}{l}\text { Grupo de tratamiento: } \\
\text { Menor experiencia }\end{array}$ & \multirow[b]{2}{*}{-0.1597} & \multirow[b]{2}{*}{$0.0816^{* *}$} & \multirow[b]{2}{*}{-0.1793} & \\
\hline & $\begin{array}{l}\text { Grupo Control: Mayor } \\
\text { experiencia }\end{array}$ & & & & $0.0503^{* * *}$ \\
\hline
\end{tabular}

Fuente: elaboración propia. ${ }^{* * *}$,** * indican nivel de significación del 1\%,5\% y $10 \%$ respectivamente.

Los resultados obtenidos se sintetizan en la figura 2, donde es posible observar cómo ciertas características personales y del entorno organizacional pueden inclinar la balanza hacia la preferencia por un tipo de estructura de liderazgo basado en la teoría de agencia o la teoría de servidores. 
Figura 2

Influencia de características personales y entorno organizacional

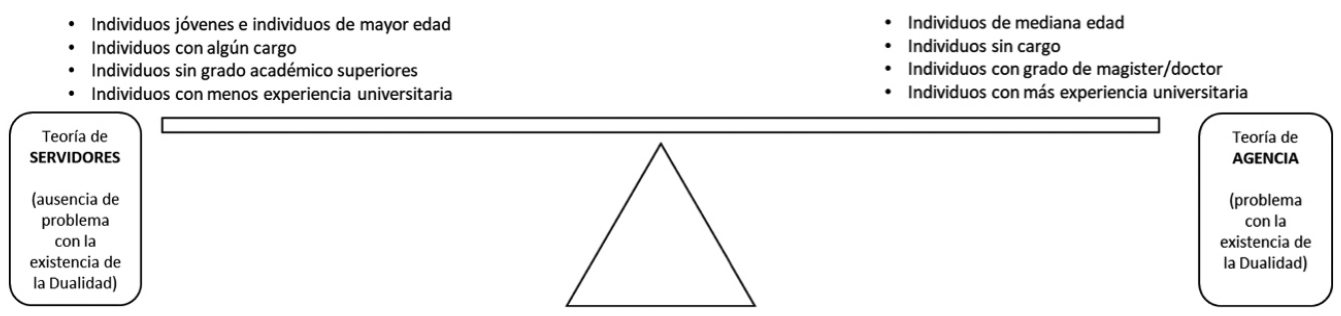

Fuente: elaboración propia.

\section{Conclusiones}

Tal como se indicó en secciones anteriores, la preferencia de los expertos por el tipo de estructura de liderazgo de los gobiernos corporativos universitarios, es decir, la preferencia por quien debe presidir el máximo cuerpo colegiado, es mayoritariamente la del tipo unitario, es decir que quien preside el MCC sea una persona distinta a la del rector. Sin embargo, se demostró que esta preferencia está condicionada de acuerdo a las características personales y del entorno organizacional de los individuos. En este sentido, nuestra hipótesis central del trabajo se confirma puesto que ambas dimensiones influyen en las preferencias. No obstante, no todas las variables incorporadas en este trabajo explicaron las preferencias por una estructura de liderazgo descrita por la teoría de agencia o la de servidores, como lo son las variables sexo y el tipo de organización (pública o privada) a la que pertenecen los expertos.

Las fortalezas de este trabajo pasan tanto por el número de observaciones utilizado como por las metodologías adoptadas, que en el caso del emparejamiento de datos (matching) no es frecuente encontrarlo en este tipo de investigaciones.

En relación a los resultados obtenidos, del lado de las características personales, se observó que expertos con mayores niveles de formación prefieren una estructura de liderazgo del tipo unitario (mayor prevalencia hacia la teoría de agencia). Al parecer esto está alineado a las características de un cuerpo académico o de gestión de las universidades, por cuanto éstas resultan organizaciones que deberían ser por excelencia lugares en el que impere la reflexión, la crítica y la transparencia en diversas dimensiones. 
En el caso de los años, se concluye que individuos de mediana edad son más críticos con la dualidad. Dada la información disponible, no hay claridad de las razones del resultado, sin embargo, podría estar dado en que los individuos más jóvenes son menos críticos; quizá las razones vayan por el hecho de estar aún alejados de la posibilidad de influir en las organizaciones o porque ven en una estructura de liderazgo del tipo dual una mayor probabilidad de desarrollo personal al existir una solo persona que pueda tomar decisiones más rápidas y contribuir a su desarrollo personal. En el caso de los académicos o profesores de más alto grupo etario, que también prefieren una estructura dual, las razones quizás estén por el hecho de que ellos están más cerca del momento en que abandonarán las instituciones.

Por el lado de la influencia del entorno organizacional, se observa que los años de experiencia en las universidades influyen sobre la preferencia por el tipo de estructura de liderazgo unitario en los gobiernos corporativos universitarios (tendencia hacia la teoría de agencia). Dicho de otro modo, mientras más experiencia, se tiende a ser más crítico con la dualidad. A su vez, la posición jerárquica dentro de las universidades juega un importante papel sobre la posición crítica frente al doble rol MCC, ya que individuos con cargos superiores e intermedios, es decir quienes toman o están cercanos al proceso de toma decisiones, son mucho menos críticos y por lo tanto más propensos a la teoría de servidores, lo que seguramente es explicado por la necesidad de tener menos obstáculos para ejercer el poder y liderazgo, también para tomar decisiones de forma más rápida y confiar mucho en sus propias capacidades directivas.

Como se podrá deducir, algunas de las características aquí analizadas no son estáticas y por lo tanto la percepción resulta dinámica a través del tiempo. De un lado, la experiencia universitaria y la edad por si misma van cambiando en el tiempo; además, los cuerpos académicos universitarios tienden a ir aumentando sus grados académicos. No obstante, el hecho de asumir cargos directivos y superar ciertos umbrales de edad tenderán a hacer a los individuos menos críticos con la dualidad.

Las implicancias teóricas de este trabajo no pasan por indicar cuál de las dos teorías es la más correcta, sino más bien por reflejar empíricamente que las características y el entorno de los cuerpos académicos de las universidades, desde el punto de vista de este trabajo, influyen y son determinantes para preferir algún tipo de estructura liderazgo en los MCc de las universidades.

Otra implicancia de los resultados, pero que no es un problema tratado en este trabajo, es desde la perspectiva del desarrollo organizacional de las universidades. Las características del cuerpo académico y de la misma organización pueden ejercer presión o no hacia un cambio de la estructura de 
liderazgo de los MCc, como también puede provocar mayor o menor resistencia organizacional a una imposición, por ejemplo, por la vía de la regulación de las leyes, del cambio del tipo de estructura liderazgo de los Mcc. En este sentido, este trabajo provee de evidencia de características que deben ser consideradas para potenciales intentos de cambios en el tipo de estructura de liderazgo al interior de las universidades.

No obstante lo anterior, se debe recordar un hallazgo no tratado en este trabajo, y es el hecho de que del cuerpo académico que actualmente en su universidad tiene una estructura de liderazgo unitario (basado en la teoría de agencia), tan solo el 2,91\% preferiría cambiar a un estructura dual, lo que contrasta fuertemente con el grupo estudiado en esta investigación. Lo anterior nos conduce a plantear como desafíos futuros el hecho de definir cuál sería el tipo de estructura de liderazgo en los MCC más adecuado para las universidades.

\section{Referencias}

Acar, E., y Ozkan, S. (2017). Corporate governance and provisions Under IAS37. Euromed Journal of Business, 12 (1), 52-72.

Adrian, C.; Wright, S., y Kilgore, A. (2016). Adapative congoint analysis: A new approach to defining corporate governance. Corporate Governance: An International Review, 1-(13).

Alfraih, M. (2016). The effectiveness of board of directors' characteristics in mandatory disclosure compliance. Journal of Financial Regulation and Compliance, 24 (2), 154-176.

Amran, A.; Periasamy, V., y Zulkafli, A. H. (2014). Determinants of climate change disclosure by developed and emerging countries in Asia Pacific. Sustainable Development, 22 (3), 188-204.

Appuhami, R., y Bhuyan, M. (2015). Examining the influence of corporate governance on intellectual capital efficiency evidence from top service firms in Australia. Managerial Auditing Journal, 30 (4-5), 347-372.

Arora, A., y Sharma, C. (2016). Corporate governance and firm performance in developing countries: Evidence from India. Corporate Governance-The International Journal of Business in Society, 16 (2), 420-436.

Ashwin, A.; Krishnan, R., y Rejie, G. (2015). Family firms in India: Family involvement, innovation and Agency and Stewardship behaviors. Asia Pacific Journal of Management, 32 (4), 869-900.

Briano-Turrent, G., y Saavedra-Garcia, M. (2015). La composición del consejo de administración y la escritura accionaria como factores explicativos de la transparencia en el gobierno corporativo en Latinoamerica: evidencia en empresas cotizadas de Argentina, Brasil, Chile y México. Estudios Gerenciales, 31, 275-286. 
Broye, G.; Francois, A., y Moulin, Y. (2017). The cost of cEO duality: Evidence from French leadership compensation. European Management Journal, 35 (3), 1-15.

Carmona, P.; De-Fuentes, C., y Ruiz, C. (2016). Risk disclosure analysis in the corporate governance annual report using. Fuzzy-set qualitative comparative analysis. RAE- Revista de Administracao de Empresas, 56 (3), 342-352.

Chancharat, N.; Krishnamurti, C., y Tian, G. (2012). Board structure and survival of new economy IPO firms. Corporate Governance: An International Review, 20 (2), 144-163.

Christensen, J.; Kent, P.; Routledge, J., y Stewart, J. (2015). Do corporate governance recommendations improve the perfomance and accountability of small lested companies? Accounting and Finance, 55, 133-164.

Dalton, D., y Aguinis, H. (2013). Measurement Malaise in strategic management studies: The case of corporate governance research. Organizational Research Methods, 16 (1), 88-99.

Das, A., y Dey, S. (2016). Role of corporate governance on firm performance: a study on large Indian corporations ofter implementation of companies Act 2013. Asian Journal of Business Ethics, 5 (1), 149-164.

Davis, J.; Schoorman, D., y Donaldson, L. (1997). Toward a stewardship theory of management. Academy of Manegement, 22 (1), 20-47.

Duru, A.; Raghavan, I., y Zampelli, E. (2016). The dinamic relationship between CEO duality and firm performance: the moderating role of board independence. Jounal Business Research, 4269-4277.

Eddleston , K.; Kellermanns, F., y Zellweger, T. (2012). Exploring the Entreprencurial Behavior of family Firms: Does the stewardship Perspective Explain Differences? Entrepreneurship Theory and Practice, 347-367.

Efron, B., y Tibshirani, R. (1986). Bootstrap Methods for Standard Errors, Confidence Intervals, and Other Measures of Statistical Accuracy. Statistical Science, 1, 54-77.

Elsayed, K. (2010). A Multi-Theory perspective of board leadership structure: What does the Egyptian corporate governance context tell Us? British Journal of Management, 21, 80-99.

Fama, E. (1980). Agency Problems and the Theory of the Firm. Journal of Political Economy, 88 (2), 288-307.

Ganga, F. (2016). Opiniones de expertos respecto del papel de rectores en los máximos cuerpos colegiados de sus universidades: presentación de avances investigativos. En F. Ganga, y J. Abello, Gobernanza Universitaria, aportes desde una Perspectiva Latinaometicana (págs. 187-201). Santiago de Chile: Ril Editores.

Ganga, F., y Burotto, J. (2012). Dimensión de los Máximos Cuerpos Colegiados de las universidades de Chile: Una mirada de agente y principal. Revista de la Educación Superior, XLI (158), 47-66.

Ganga, F., y Maluk, S. (2016). Opiniones de expertos sobre el doble rol en las universidades ecuatorianas. Revista Educacao, Ciencia e Cultura, 21 (2), 57-73.

Gao, N., y Jain, B. (2012). Founder Management and the market for corporate control for IPO Firms: The moderating effect of the power structure of the firm. Journal of Business Venturing, 112-126. 
Gaur, S., Bathula, H., y Singh, D. (2015). Ownership concentration, board characteristics and firm performance a contingency framework. Management Decision, 53 (5), 911-931.

Guillet, B.; Seo, K.; Kucukusta, D., y Lee, S. (2013). CEO duality and firm performance in the U.S. restaurant industry: Moderating role of restaurant type. International Journal of Hospitaly Management, 33, 339-346.

Kolb, D.; Rubin, I., y McIntyre, J. (1980). Psicología de las organizaciones, problemas contemporáneos. Madrid, España: Editorial Sossat S.A.

Krause, R. (2017). Being the CEO's boss: an examination of board chair orientations. Strategic Management Journal, 38 (3), 697-713.

Lai, L., y Tam, H. (2017). Corporate governance, ownership structure and managing earnings to miet critical thresholds among Chineses listed firms. Review of Quantitative Finance and Accounting, 48 (3), 789-818.

Levin, R., y Rubin, D. (2010). Estadística para administración y economía. Séptima Edición, Naucalpan de Juárez, México: Pearson Educación.

Li, F.; Li, T., y Minor, D. (2016). CEO power, corporate social responsability, and firm value: a test of agency theory. International Journal of Managerial Finance, 12 (5), 611-628.

McGuinness, P.; Lam, K., y Vieito, J. (2015). Gender and Other major board characteristics in China: explaining corporate dividend policy and governance. Asia Pacific Journal of Management, 32 (4), 989-1038.

Nas, T., y Kalaycioglu, O. (2016). The effects of the board composition, board size and CEO duality on export performance: Evidence from Turkey. Management Research Review, 39 (11), 1374-1409.

Perez-Calero, L.; Guerrero-Villegas, J., y Hurtado, J. (2017). The influence of organizational factors on board roles. Management Decision, 55 (5), 842-871.

Petrou, A., y Procopiou, A. (2016). CEO shareholdings and earnings manipulation: A behavioral explanation. European Management Review, 13 (2), 137-148.

Pfeffer, J. (1993). El poder en las organizaciones: política e influencia en una empresa. Madrid-España: McGraw-Hill.

Pham, N. L.; Oh, K. B., y Pech, R. J. (2015). Mergens and acquisitions: CeO duality, operating performance and stock returns in Vietnam. Pacific-Basin Finance Journal, 35, 298-316.

Qian, C.; Wang, H.; Geng, X., y Yu, Y. (2017). Rent appropriation of knowledgeBased assets and firm performance when institutions are weak: An study of Chinese publicly listed firms. Strategic Management Journal, 38 (4), 892-911.

Rahman, A., y Hamdam, M. (2017). The extent of compliance with FRs 101 standard: Malaysian evidence. Journal of Applied Accounting Research, 18 (1), 87-115.

Rao, K., y Tilt, C. (2016). Board diversity and csR reporting: An Australian study. Meditari Accountancy Research, 24 (2), 182-210.

Rodrigues, L. L. (2017). Corporate governance and intellectual capital reporting in a period of financial crisis: evidence from portugal. International Journal of Disclosure and Governance, 14 (1), 1-29.

Ruiz, J. (1995). Sociología de las organizaciones. Bilbao: Universidad de Deusto. 
Schepker, D., y Oh, W. Y. (2013). Complementary or substitutive effects? Corporate governance mechanisms and Poison Pill Repeal. Journal of Management, 39 (7), 1729-1759.

Sheikh, N., y Khan, M. (2016). Effects of excess cash, board attributes and insider ownership on firm value: evidence from Pakistan. Australasian Accounting Business And Finance Journal, 10 (1), 29-39.

Tang, J. (2017). CEO duality and firm performance: The moderating roles of other executives and blockholding outside directors. European Management Journal, 35 (3), 362-372.

Taniman, C., y Shannassy, T. (2015). Exploring the influence of chief executive officer professional development and work context on organisation performance: A multi-theoretic perspective. Journal of Management $\mathcal{E}$ Organization, 21 (5), 675-694.

Tien, C.; Chen, C.-N., y Chuang, C.-M. (2013). A study of CeO power, pay structure and firm performance. Journal of Mangement \& Organization, 19 (4), 424-453.

Titova, Y. (2016). Are board characteristics relevant for banking efficiency? evidence from the US. Corporate Governance-The International Journal of Business in Society, 16 (4), 655-679.

Torchia, M., y Calabro, A. (2016). Board of directors and financial transparency and disclosur. Evidence from Italy. Corporate Governance-The International Journal of Business in Society, 16 (3), 593-608.

Veprauskaite, E., y Adams, M. (2013). Do powerful chief executives influence the financial performance of UK firms. The British Accounting Review, 45 (3), 229-241.

Villanueva-Villar; M., Rivo-López, E., y Lago-Peñas, S. (2016). On the relationship between corporate governance and value creation in an economic crisis: Empirical evidence for the Spanish case. Business Research Quarterly, 19, 233245.

Wagner, J. (2007). What a Difference a Y makes-Female and Male Nascent Entrepreneurs in Germany. Small Business Economics, 28, 1-21.

Wang, D.; Sun, D.; Yu, X., y Zhang, Y. (2014). The impact of CEO daulity and ownership on the relationship between organisational slack and firm perfomance in China. Systems Research and Behavioral Science, 94-101.

Withers, M., y Fitza, M. (2017). Do board chairs matter? the influence of board chairs on firm performance. Strategic Management Journal, 38, 1343-1355.

Zarco, V.; Rodriguez, A., y Martón, M. (2004). Introducción a la psicología de las organizaciones, la psicología en el estudio de las organizaciones y la conducta laboral. En A. Rodriguez, Psicología de las organizaciones (págs. 19-67). Barcelona, España: UOC. 
Anexo 1

Selección de preguntas desde el cuestionario: Estudio sobre gobierno universitario (proyecto Conicyt, Fondecyt/Regular $N^{\circ} 1131134$ )

\begin{tabular}{|c|c|}
\hline Dimensión & Descripción \\
\hline Pregunta (sin número) & $\begin{array}{l}\text { Sexo } \\
\begin{array}{ll}\text { a. Masculino } & \text { b. Femenino }\end{array}\end{array}$ \\
\hline Pregunta 1 & Universidad/Institución: \\
\hline Pregunta 2 & Cargo en la Universidad/Institución: \\
\hline \multirow[t]{2}{*}{ Pregunta 4} & Años de trabajo en la universidad/Institución: \\
\hline & Rango de edad \\
\hline Pregunta 5 & $\begin{array}{lll}\text { a. Menor de } 35 \text { años } & \text { b. De } 35 \text { a } 45 \text { años } & \text { c. De } 46 \text { a } 60 \text { años } \quad \text { d. Mayor de } 60 \text { años }\end{array}$ \\
\hline Pregunta 6 & $\begin{array}{l}\text { Titulo profesional: } \\
\text { Grado: } \\
\text { Post grado(s): }\end{array}$ \\
\hline Pregunta 12 & $\begin{array}{l}\text { ¿Quién es el Presidente del MCC de su Universidad? } \\
\begin{array}{ll}\text { a. El Rector b. Un integrante elegido por los miembros del MCC } \\
\text { c. El integrante más antiguo del MCC d. Otro (menciónelo): }\end{array}\end{array}$ \\
\hline Pregunta 13 & $\begin{array}{l}\text { De acuerdo a su punto de vista ¿Quién debiera ser el Presidente del MCC de su } \\
\text { Universidad? } \\
\begin{array}{ll}\text { a. El Rector b. Un integrante elegido por los miembros del MCC } \\
\text { c. El integrante más antiguo del MCC d. Otro (menciónelo): }\end{array}\end{array}$ \\
\hline
\end{tabular}


